\title{
Politique
}

\section{Une relecture de John F. Kennedy}

\section{Pierre Laurin}

Volume 1, numéro 1, janvier 1982

Les intellectuels et les pouvoirs

URI : https://id.erudit.org/iderudit/040393ar

DOI : https://doi.org/10.7202/040393ar

Aller au sommaire du numéro

Éditeur(s)

Société québécoise de science politique

ISSN

0711-608X (numérique)

Découvrir la revue

Citer cet article

Laurin, P. (1982). Une relecture de John F. Kennedy. Politique, 1(1), 73-88.

https://doi.org/10.7202/040393ar

Ce document est protégé par la loi sur le droit d'auteur. L'utilisation des services d'Érudit (y compris la reproduction) est assujettie à sa politique d'utilisation que vous pouvez consulter en ligne.

https://apropos.erudit.org/fr/usagers/politique-dutilisation/
Cet article est diffusé et préservé par Érudit.

Érudit est un consortium interuniversitaire sans but lucratif composé de l'Université de Montréal, l'Université Laval et l'Université du Québec à Montréal. Il a pour mission la promotion et la valorisation de la recherche. https://www.erudit.org/fr/ 


\title{
Une relecture de John F. Kennedy
}

\author{
Pierre Laurin \\ Université de Montréal
}

Pour plusieurs personnes de la génération qui a aujourd'hui vingt-cinq ans, John F. Kennedy est l'homme qui est mort au combat.

À l'étude, il devenait encore plus séduisant: il accéléra la déségrégation scolaire, lutta contre le trust de l'acier, consacra l'émancipation des Noirs, etc... Rappelons-nous aussi son style flamboyant, son «sex-appeal», son attitude frondeuse (imitée plus tard par plusieurs autres chefs politiques). La nouvelle de sa mort eut l'effet d'une bombe; pour plusieurs, elle a été le résultat d'un complot ourdi par des forces de droite qui craignaient ce président jeune et supposément ouvert à de nouvelles idées. Tapis dans l'ombre, les comploteurs auraient ainsi mis un terme à une présidence qui était potentiellement celle du changement, celle annonciatrice d'une nouvelle voie pour l'Amérique. C'est à tout le moins le témoignage de James Hepburn dans Farewell America (Frontiers 1968). Dans un texte parfois délirant de nostalgie, l'auteur nous décrit comment les gens de sa génération ont vu la mort de Kennedy. Bien qu'intéressant, ce livre a quand même une carence de taille; l'objectivité. D'autres auteurs plus articulés émettent un autre son de cloche. 
À leur lecture, nous avons essayé de découvrir les postulats que John F. Kennedy couvait d'une éloquence si puissante. L'étude de la politique étrangère nous semblait indiquée. Je convie le lecteur à l'étude des hauts faits des États-Unis sur la scène mondiale pour tirer des conclusions; le «missile gap", l'intervention au Vietnam, le sommet de Vienne, etc...

Cette démarche nous conduira à réévaluer nos souvenirs d'enfants: ce leader charismatique n'a peut-être pas été aussi libéral et évolué qu'on l'aurait cru. Il faut au demeurant lui retirer l'étiquette progressiste dont on l'affuble trop souvent. En fait, il faut redécouvrir complètement cet homme. Issu de l'après-guerre, ayant grandi dans la rhétorique anticommuniste, il était juste un peu plus modéré que Kennan et Joe Mc Carthy.

Comme pour définir ses priorités, le discours inaugural de John F. Kennedy porte presque uniquement sur la politique étrangère. Dans une allocution souvent citée par les historiens, le jeune président propose le renouveau. Kennedy suggère au peuple américain un rôle international plus agressif, plus présent. En jetant un regard critique sur le passé, (surtout sur l'administration Eisenhower) il veut refaire des États-Unis le champion, à l'échelle planétaire, de la liberté des peuples et redonner au pays une prééminence qu'il disait perdue.

Plusieurs contemporains sont pourtant très critiques face à ce discours martial: Henrie Fairlie remarque, face à ce néoimpérialisme, que «le peuple américain fut persuadé qu'il pouvait être le gardien des remparts de la liberté dans le monde, goûtant le sentiment d'appartenance à un empire, exalté par la mission qu'il se donnait ${ }^{1}$.

D'un autre côté, on ne peut nier l'importance du mythe Kennedy car «sa jeunesse, les circonstances tragiques de sa

1. Henrie Fairlie. The Kennedy Promise, Double day \& Co., New York 1973. p. 231 Notre traduction. 
mort, ont servi d'éléments aux médias pour son amplification ${ }^{2}$. Cette légende a amené à titre posthume, si je puis dire, des appréciations positives de la politique étrangère des ÉtatsUnis de l'époque.

C'est dans cette polémique que s'inscrit notre contribution: John F. Kennedy se démarque-t-il favorablement de ses prédécesseurs? Donne-t-il un nouveau visage, plus acceptable, à la politique étrangère américaine et inspire-t-il ses successeurs? Un examen rapide et bref des événements marquants de cette période nous pourvoiera des outils nécessaires à une évaluation.

Jeune homme fougueux et érudit, Kennedy est critique de la présidence d'Eisenhower. Se servant de la théorie du pendule développée par Arthur M. Schlesinger (père et fils), il oppose à un passé défaitiste un avenir ambitieux. Un discours habile, développé pendant sa campagne contre Nixon, prête à l'administration en place un laxisme qui aurait donné la suprématie mondiale à l'U.R.S.S. Le but qu'il se donnait: restituer au pays la primauté. «A long era of somnolescence seemed over» disait Walter Lippmann ${ }^{3}$.

Le «missile gap»:

Dès les premiers jours de sa campagne, Kennedy veut montrer la supposée faiblesse des États-Unis : il évoque le «missile gap». Pendant les années Eisenhower, l'Union Soviétique aurait réussi à dépasser les Américains au niveau de l'armement nucléaire. Selon Russel F. Weigley ${ }^{4}$, cette psychose serait le résultat de la supériorité notoire des Soviétiques au niveau spa-

2. Edmond Orban. La présidence moderne aux États-Unis. Presses de l'Université du Québec à Montréal. Montréal. 1979. p. 46.

3. Ronald Steel. Walter Lippmann and the American Century. Little Brown \& Atlantic. Boston 1980. p. 525 (notre traduction).

4. Russel F. Weigley. The American W'ay of War. Mc Millan \& Co.. New York 1973. p. 441. 
tial (c.f. Spoutnik 1957). En 1961, le secrétaire à la Défense, Mc Namara, s'empresse de rassurer la population: les propos du président étaient sans fondements. Malgré cela, le budget de la défense (et surtout le programme des missiles) gonfle considérablement. Laissons Raymond Aron résumer cette question: «L'équipe Kennedy a fait, dès son arrivée à la MaisonBlanche, un effort considérable non pour combler l'écart en fait d'engins (écart qui n'a jamais existé) mais pour s'assurer une incontestable supériorité » 5 .

Ainsi, on peut tout de suite établir le postulat suivant: Kennedy réclame une «présidence armée » et renie l'héritage du général Eisenhower en mettant un terme aux gestes lenifiants envers l'U.R.S.S.. D'autres événements montrent avec acuité cette attitude de grande fermeté du président.

\section{La Baie des Cochons:}

Respectant sa promesse de fermeté envers Cuba, Kennedy permet la réalisation d'un plan imaginé sous la présidence d'Eisenhower: faire tomber Castro en profitant du mécontentement populaire. Le fer de lance de cette attaque était assuré par le débarquement, au nord de l'île, d'anti-castristes entraînés au Guatemala par la C.I.A.. Castro réplique avec force, repousse l'attaque et mâte les quelques insurgés. Devant cette faillite totale, Kennedy refuse l'escalade; tournant casaque, il interdit le soutien aérien qui aurait été nécessaire à la continuation de l'opération.

Comme il fallait s'y attendre, s'ensuit un tollé de protestations. D'abord de Castro qui stigmatise les États-Unis pour la violation du territoire cubain. Ensuite l'U.R.S.S. qui dénon-

5. Raymond Aron. Le grand débat: initiation à la stratégie atomique. CalmanLévy. Paris 1973. p. 83. 
ce ce geste impérialiste et remet en question les discussions sur la détente. Mais les condamnations viennent aussi du pays.

La plupart des critiques ont questionné le jugement du président prêt à suivre ainsi un plan mis au point par ses prédécesseurs. D'un même souffle pourtant, ils louent sa prudence et approuvent son refus de l'escalade. En revanche, les délateurs sont intraitables envers ce qu'ils appellent les vrais responsables de cette folle escapade: les hauts fonctionnaires de la C.I.A. et du département d'État hérités de l'ancienne équipe. Ainsi, Walter Lippmann conclut qu'une «faute d'une telle importance ne peut être effacée que par la démission des personnes-clés qui sont vraiment responsables ${ }^{6}$. Le président profita de ce que le blâme éclaboussa plusieurs officiels. Or, il faudrait avoir l'imagination d'un romancier pour croire que Kennedy n'était pas au courant de tous les détails de l'opération: déjà cinq mois avant son entrée à la Maison-Blanche, des bruits circulaient sur l'existence d'un tel plan. Il aurait pu réagir vivement et mettre un terme aux préparatifs. Il ne l'a pas fait.

Il était clair, dès son entrée à la Maison-Blanche, que Kennedy recourerait à des interventions armées pour prouver la force des États-Unis. Il ne voyait les relations avec l'U.R.S.S. qu'à travers le prisme de l'affrontement, du coup de main et de la violence. Selon David Halberstam, il voulait tenir la dragée haute aux Russes et aux Cubains; il était déterminé «à montrer sa valeur...; à être capable de tenir tête aux Soviétiques, et prouver qu'il était aussi dur qu'eux, aussi prompt à dégainer ${ }^{7}$.

6. Cité par R. Steel, op. cit. . p. 529 (notre traduction).

7. David Halberstam. On les disait les meilleurs et les plus intelligents. Ed. Robert Laffont. Paris 1972, p. 91. 


\section{Le sommet de Vienne:}

Cette rencontre, qui devait donner aux chefs d'État l'occasion de calmer un peu les tensions, a plutôt été l'occasion de les accroître.

Dès les premiers échanges, Kroutchev tient un langage agressif, provoquant même. Il menace de lancer des tanks sur Berlin et de déchainer l'apocalypse nucléaire si les États-Unis récidivent à Cuba. Kennedy, stoïque, répond tout aussi brutalement. Le sommet est plus annonciateur d'affrontements orageux que de négociations pacifiques.

Il était certain que Kroutchev serait acerbe et violent lors de la rencontre. L'ambassadeur itinérant Harriman qui, trois semaines plus tôt, avait visité Kroutchev en Russie, savait pourtant que derrière ce discours dur et intraitable existait un désir de paix et de coexistence pacifique. Kennedy ignore les propos de Harriman et contribue, avec Kroutchev, à augmenter la tension entre les deux pays.

Des discussions de Vienne, Kennedy tire une leçon: il doit prouver aux Russes la puissance américaine. À la toute fin de la conférence, il aurait dit à l'éditeur du New York Times «si nous avons un problème pour faire croire à notre puissance, le Vietnam me semble un bon endroit pour ça ${ }^{8}$. Cyniquement, volontairement, Kennedy pousse plus avant l'Amérique dans l'aventure vietnamienne.

\section{L'implication au Vietnam:}

De deux mille «conseillers militaires» américains au Vietnam en 1961, on passe en 1963 à quinze mille cinq cent. Pourquoi cette escalade? Qu'est-ce qui explique cette implication si aberrante à prime abord? Comme nous l'avons signalé

8. Ibid.. p. 196. 
il y a là une préoccupation de montrer sa force. De plus, on peut noter l'importance d'impératifs électoraux; il est clair que Kennedy veut un succès sur la scène internationale. Pendant les années ' 61 et ' 62 , les événements qui ont secoué le monde ne lui ont donné que des victoires mitigées: Berlin, la crise des missiles, la neutralisation du Laos, etc... Le Vietnam semblait être la dernière chance et il fallait, selon lui, jouer cette carte. L'historien Weigley illustre bien le problème ${ }^{9}$.

Pourtant, une question se pose, comment ce jeune président, féru d'histoire, pouvait-il croire à la réussite de l'opération quand les Français, avec plus de deux cent mille hommes, n'avaient pu mâter les insurgés vietnamiens? Il connaissait bien l'exemple de Mao qui, avec des hommes mal équipés (mais profitant de l'essentiel appui des paysans), avait vaincu une armée mieux outillée et plus nombreuse. Kennedy s'implique car il est sûr de gagner, malgré les précédents. Comme première raison, le président s'inscrivait dans la continuité de la croisade anticommuniste. Adoptant une vision manichéenne surannée, il fustige les rebelles de la terre en les assimilant à des marionnettes communistes. Il est donc pour lui légitime de les combattre. Rappelons-nous, deuxièmement, que Kroutchev avait promis son support à tout mouvement de libération nationale.

S'inspirant de Che Guevara et de Mao Tse Toung, il comprend l'importance de la guérilla. Il ordonne la création d'un corps d'élite (les bérêts verts) entraînés pour faire face aux communistes du Nord-Vietnam. Dès 1961, des contingents sont envoyés en Asie du sud-est avec mission de «conseiller». Or, la réalité devient toute différente: l'armée du Sud-Vietnam est conventionnelle et ignore les raffinements anti-guérilla venus d'Amérique. Ainsi les troupes américaines sur place arrêtent de conseiller et en viennent vite à commander. Le vœu de Kenne-

9. Russel F. Weigley, op. cit., p. 452. 
dy de voir cette guerre faite et gagnée par les Sud-vietnamiens se démarque de plus en plus de la réalité. L'engrenage est enclenché; l'escalade confronte les dirigeants américains.

L'histoire nous apprend que dès 1961, les troupes sudvietnamiennes enregistrent défaites sur défaites. Comment se fait-il que les rapports remis au président parlent de victoires éclatantes? Les conseillers du président et les militaires sur le terrain se chargeront de maquiller la réalité. Notons surtout l'influence de Maxwell Taylor, conseiller spécial du président pour les affaires militaires qui obtient, pour son ami Harkins, le commandement des forces militaires américaines au Sud-Vietnam. Selon l'étude pénétrante de David Halberstam précédemment citée, Taylor aurait demandé à Harkins de teinter d'optimisme les rapports envoyés au pays.

Ainsi, le président croyait fermement que la victoire était imminente. Mais comment se fait-il que l'équipe présidentielle devint si impénétrable à toute dissonance? Pourquoi ostracisat-on les sceptiques? Selon Henrie Fairlie, cela tient à l'unanimité des conseillers du président. Ce dernier ignora le style de Roosevelt qui s'entoura de gens différents, d'adversaires parfois, s'inspirant du résultat de leurs oppositions.

Ainsi, Robert Kennedy rassure son frère sur la victoire qu'il croit prochaine. Mc Namara à la Défense cautionne tous les gestes du président. Ancien président de la Compagnie Ford, Mc Namara vit au rythme des ordinateurs et prend en charge les opérations au Vietnam. "Évaluant» mathématiquement la situation, armé de cartes et de statistiques optimistes, il prévoit que les petits hommes bruns du Vietnam seront rapidement défaits. Rusk, le secrétaire d'État, quoique discret dans cette affaire, ne fait que joindre sa voix au concert.

Bientôt pourtant, le discours rassurant de ce cénacle prête flanc à la critique. Témoins et détracteurs de plus en plus crédibles, les journalistes américains en poste au Vietnam en 
viennent à semer le doute. David Halberstam, envoyé par le New York Times se gagne un Pulitzer pour la qualité et la pertinence de ses textes. Comme ses confrères, il ramène le pays à la dure réalité. Les doutes de Kennedy sont confirmés. Les voyages de Mc Namara sur place cautionnent les dénonciations des reporters. Des têtes roulent, notamment celle de Harkins. Les civils réussissent à percer le mur des rapports militaires trop «optimistes».

L'unanimité étant rompue, une brèche est ouverte dans l'aéropage de faucons qui entoure le président. Harriman, vieillard de soixante-dix ans, se fait enfin écouter et devient soussecrétaire d'État. Helsman en est un autre qui s'oppose à la guerre au Vietnam; on le nomme sous-secrétaire aux affaires d'Extrême-Orient.

Mais malgré la connaissance de cette nouvelle réalité, la machine est lancée et semble difficile à arrêter. Selon Sorensen, Kennedy ne voulait pas envoyer d'hommes au Vietnam; c'est sous les pressions de ses conseillers qu'il y aurait consenti ${ }^{10}$. L'historien militaire Weigley, lui, dit que l'implication militaire doit être imputée au président : «un président qui a choisi, comme style pour son administration, une image de force seulement comparable à celle qu'a voulu se donner à l'époque Théodore Roosevelt ${ }^{11}$. On sent bien ici que le militaire veut protéger ses pairs, rejetant le poids de l'erreur sur les seules épaules de Kennedy.

Au milieu de l'année 1962, on devient sceptique. David Halberstam va plus loin: «Tous ... savaient qu'on était en train de perdre la guerre, mais ils n'exprimèrent jamais cette idée sur le papier ni dans leurs déclarations ${ }^{12}$. Kennedy était per-

10. Théodore C. Sorensen. Kennedy. Mc Millan \& Co.. 1965. p. 654 (notre traduction).

11. Russel F. Weigley. op. cit. . p. 460 (notre traduction).

12. Halberstam. $n$. cit. p. 323. 
plexe. L'échéance de l'élection présidentielle lui dictait de taire son incrédulité. Une fois réélu, aurait-il engagé un retrait des troupes? Question intéressante que sa mort laisse sans réponse.

Une chose est certaine pourtant: Kennedy et ses conseillers ont amené le Gulliver américain à aller s'empêtrer dans les marais vietnamiens. Véritable hécatombe pour toute une jeunesse, cette "escapade» ne mérite pas de l'histoire une glorieuse épitaphe.

La crise des missiles:

Événement charnière s'il en est, cette crise redore le blason du président au niveau international.

Pendant plus d'un mois (18 octobre au 20 novembre 1962) le déchaînement de l'apocalypse nucléaire guette le monde. Nikita Kroutchev ordonne l'installation de rampes de lancement pour missiles nucléaires en sol cubain. Des photos, prises d'un avion-espion américain (U-2) montrent ces rampes pointées vers l'Amérique. Croyant que le fort trafic maritime entre I'U.R.S.S. et Cuba permet l'arrivée de missiles à têtes nucléaires potentiellement meurtrières pour les États-Unis, Kennedy ordonne un blocus maritime. Un "cordon sanitaire» entoure Cuba et tout navire russe le franchissant s'expose à une réplique armée. Malgré cette violation manifeste du droit international de la mer, les Russes obtempèrent et tournent bride. Les duellistes passent à un cheveu de l'affrontement.

Malgré le fait qu'il pratique le «eyeball to eyeball diplomacy", le président provoque l'unanimité dans le pays. Ainsi, les maquignons des deux côtés conviennent du compromis suivant: retrait des missiles contre l'impunité de Cuba. Cette presque victoire redonne confiance à Kennedy.

La frousse générale causée par le sentiment d'être au bord du précipice a eu un effet lénifiant sur les relations entre les 
deux pays. Encore plus conscients des dangers du conflit nucléaire, les leaders des deux pays acceptent de s'asseoir à la même table pour négocier les modalités de gestes concrets de détente.

Le premier pas (petit peut-être mais réel) est la signature à Genève en juin 1963 du traité sur la limitation des essais nucléaires souterrains. Les premiers jalons de la détente sont posés.

D'autres indices permettent de dire que certaines percées de soleil crèvent le ciel sombre des relations U.S.A./U.R.S.S. . Soulignons notamment un discours de Kennedy en 1963 à l'O.N.U. qui fait appel à une coopération spatiale, pouvant aller jusqu'à une mission conjointe sur la lune. En octobre, le président permet la négociation d'accords sur la vente de blé.

Des gestes timides certes, mais annonciateurs d'une période de rapprochement et de détente entre les deux pays.

Attitude face aux pays du tiers-monde:

En ce domaine, le président s'est donné d'imposants objectifs. Dans ce qu'il appelle la «Decade for development», il promet aux pays pauvres l'aide des États-Unis. Ainsi, il prend conscience que les «États-Unis ne sont ni omnipotents ni omniscients $(\ldots)$ et nous ne pouvons pas imposer notre volonté à plus de $94 \%$ de l'humanité ${ }^{13}$.

Dans les premières semaines de son administration, dès le 15 mars, il annonce en Uruguay son programme «d'Alliance pour le progrès». Programme de vingt milliards de dollars étendu sur dix ans, il vise un développement général du continent sud-américain. Comme on le sait, ce fut un échec presque complet. Les dictatures et les oligarchies profitent de cette manne. Ne suggérant que des changements timides, il renforce sou-

13. William E. Leuchtenburg. The Grouth of The American Republic. Oxford University Press. New York 1980. Vol. II, p. 749. 
vent le statu quo. Henrie Fairlie explique que «personne ne peut douter que l'Alliance pour le progrès, visant au départ l'Amérique latine, se transforme bientôt en instrument de la mission impériale des États-Unis et même de manière plus directe, de sa politique étrangère ${ }^{14}$.

Mais pourquoi un échec aussi retentissant? Il y a plus que les structures des régimes politiques des pays bénéficiaires des subventions. Il faut examiner les fondements de l'économie internationale pour comprendre ce qui se passe.

Kennedy croyait que le sous-développement était causé par un manque d'opportunité de ces pays, un manque de chance. Voilà une belle expression de mentalité puritaine. Ainsi, il est prêt à apporter un soutien à qui le veut. Cette vision des choses imprègne bien les théories de Walter Rostow, un conseiller du président, qui en 1960 a écrit Stages of Economic Growth. Convaincu que les pays en voie de développement ne pouvaient se sortir de la stagnation qu'en suivant des étapes précises de progrès économique (agriculture, industrialisation, etc...), Kennedy se fait fort de pourvoir les intéressés en matériel et en ressources. Cette vision parcellaire ignore une réalité globale plus importante; que ces remèdes n'ont aucune commune mesure avec l'ampleur des problèmes des pays pauvres!

$\mathrm{Ne}$ changeant rien au niveau structurel, les programmes d'aide de Kennedy ne font souvent que systématiser le sousdéveloppement. Le Trade Expansion Act de 1962, entériné dans le cadre du Kennedy Round de 1964, en est un exemple. À la suite d'une baisse des barrières tarifaires, les pays du tiersmonde deviennent encore plus vulnérables. Les multinationales américaines se lancent encore plus fébrilement à l'assaut du monde. Ainsi, comme le reconnaît l'économiste Niveau, «le marché libre (comme le propose le Kennedy Round) a peu de

14. Henrie Fairlie, op. cit. p. 113 (notre traduction). 
vertus pour les pays pauvres et les industries naissantes qui ont besoin d'être protégés contre la concurrence étrangère " ${ }^{15}$.

Croire que l'ère de Kennedy a été celle d'une aide efficace au tiers-monde ne peut que faire sourire. Le Peace-Corps, créé par décret présidentiel, semble avoir été l'exutoire du romantisme de John F. Kennedy. Le cœur serré, il voyait ces jeunes Américains partir de par le monde vaincre la pauvreté. Edmond Orban souligne bien l'ambiguité; «la conception du rôle du Peace Corps... intéressante au départ, ne constitue cependant qu'un faible palliatif, masquant mal l'étendue des problèmes du sous-développement ${ }^{16}$. Ainsi, son attitude face au tiers-monde qui parvient difficilement à survivre peut se définir comme suit : une fumeuse contradiction qui se dissimule mal derrière l'écran de la rêverie.

La violence: matrice de la politique extérieure:

L'examen des faits nous amène, ici, à nous poser une autre question. À quoi doit-on l'utilisation quasi-systématique de la force pendant les mille jours de l'administration Kennedy? D'où vient ce besoin de montrer sa robustesse (toughness comme il le dit lui-même)? Certains diront qu'il faut chercher dans l'enfance du président. Son père, un homme dur, aurait enseigné à ses enfants le respect de la force et l'utilisation qu'on doit en faire ${ }^{17}$. D'autres plus crédibles soutiennent que la violence dans la politique étrangère américaine est une constante qui résiste aux changements de présidents. Une interprétation plus nuancée peut trouver place.

15. Maurice Niveau. Histnire des faits écnnomiques contempnrains. Presses Universitaires de France. Paris 1973. p. 633.

16. Fdmond Orban. $n$ p. cit. p. 53.

17. Soulignons notamment, à ce niveau. la contribution de Fairlie, op. cit. . pp. 69-73. 
Il faut compter avec le flair politique de Kennedy qui ne fait de gestes importants qu'en pensant à son éventuelle réélection. Comme nous le soulignionś déjà, de nombreuses crises, dont le président aurait eu vent avant qu'elles n'éclatent, auraient pu être évitées. Selon plusieurs, il aurait volontairement laisser pourrir les situations. Dans son livre, Profiles in Courage qui lui a valu un Pulitzer, Kennedy prend à témoin l'histoire et dit que «les grandes crises font les grands présidents». Il veut être un grand président et est conscient que les crises favorisent l'unanimité. C'est cynique mais efficace.

Cette quête d'accords complets ne passe pas seulement par l'exacerbation des sentiments, elle appartient aussi aux réalités politiques du pays. Se réclamant du «centre», Kennedy ne craint rien sur son flanc gauche. Les forces progressistes américaines ne sont pas vraiment articulées et ne représentent pas de poids politique réel. Pourtant, il doit ménager la droite qui, elle, au cours de l'histoire, a accumulé ses lettres de noblesse. Pour ne pas se laisser dépasser sur la droite, il pille hardiment son programme: augmentation du budget militaire, interventions armées dans le monde, etc...

Ainsi sa mise en scène pouvait laisser croire au président qu'il se présenterait avec force devant l'électorat et obtiendrait facilement un nouveau mandat présidentiel.

\section{Conclusion:}

Après ce tour d'horizon rapide des grands moments de l'administration Kennedy l'occasion semble propice à une réponse aux questions posées en introduction.

D'abord, Kennedy se démarque-t-il favorablement de ses prédécesseurs? Pour moi, non. La tête de turc préférée de Kennedy était Eisenhower qu'il dénonçait pour avoir, selon lui, laissé glisser les États-Unis au deuxième rang mondial. Or, 
Eisenhower pouvait, lui, se vanter de n'avoir jamais engagé les États-Unis dans une guerre pendant ses deux mandats. De plus, un examen rapide de cette période nous permet certes de voir un vieux général conservateur (qui semblait parfois préférer le golf à la politique), mais qui au moins a fait des gestes pacifiques concrets. Si on excepte les propos délirants de son secrétaire d'État Dulles, Eisenhower s'est présenté comme un héraut de paix. En 1953, il propose une coopération nucléaire avec l'U.R.S.S. . En 1955, il propose l' «open sky » permettant aux avions-espions des deux pays de vérifier l'emplacement des engins nucléaires sur les deux territoires. Cela aurait eu pour effet de décourager sensiblement le recours aux armes. Rappelons aussi les gestes appaisants posés en 1956 à l'occasion de la crise de Suez.

Comme on peut le voir, on est bien loin des gestes brusques, quasi suicidaires, de l'ère de Kennedy. Jusqu'à la toute fin de son mandat, le général est conséquent; son Farewell Address prévient le pays de ne pas tomber dans le piège du complexe militaro-industriel. C'est directement vers cela que Kennedy va mener les États-Unis. Quand même sage ce «vieillard-sénile »...

Répondons à la deuxième question; Kennedy inspire-t-il ses successeurs? Oui, et très clairement. Johnson reprend le flambeau de la présidence impériale, mais pas encore autant que Nixon qui continue le viol du Vietnam, même s'il veut sortir le pays de ce guépier. Après la pose post-Vietnam de l'administration Carter, on assiste aujourd'hui avec Reagan, à une inéluctable retour des choses.

Des similarités frappantes peuvent être remarquées si on compare le style de Kennedy à celui de Reagan. Cela peut avoir l'air hérétique de comparer celui que certains se plaisent encore à voir comme un «jeune progressiste» avec ce vieil acteur nostalgique. Et pourtant... 
La campagne électorale de Reagan, comme celle de Kennedy, invoquait l'infériorité militaire des États-Unis face à l'U.R.S.S.. Dans les deux cas, plusieurs s'accordent à dire que ces propos démagogiques sont des constructions de l'esprit. De plus, imitant avec vingt ans de retard John F. Kennedy, Reagan redonne vie aux "corps d'interventions rapides", ces bérêts verts des années ' $80^{18}$. En cela, il continue aussi les gestes de Kennedy.

Le nouveau président fourbit ses armes et habille la politique étrangère américaine du manteau de la violence et de l'intervention armée.

De cette manière, l'attitude tant décriée de Reagan face au Salvador n'est pas tellement différente de celle de Kennedy face au Vietnam. Le peuple américain semble pourtant moins aventureux que ses dirigeants. Dans un sondage publié dans le Time du premier juin 1981 , on se prononce à $47 \%$ pour le retrait des «conseillers militaires» du Salvador ${ }^{19}$. C'est à croire que la population tire plus de leçons de l'histoire que ne savent le faire les meneurs actuels du pays.

Devenant plus critique Walter Lippmann dit, après la mort de Kennedy, que «jamais il ne voulait être impopulaire (...). L'administration Kennedy a été un ensemble bigarré d'erreurs et de faux-départs» ${ }^{20}$. Ce témoignage de l'éminent journaliste tranche par sa pertinence.

Les mille jours montrent un Kennedy qui modernise l'arsenal du pays et systématise l'intervention impérialiste. En cela, il se démarque d'Eisenhower et inspire ses successeurs pour le pire.

18. Pour plus de détails sur cette question. voir l'article de Michel Klare «Une stratégie d'intervention sur mesure pour le tiers-monde» dans Le Monde diplomatique. Avril 1980. p. 8.

19. Cité par John F. Stacks. "Its Rightwards on». dans Time. premier juin 1981. p. 8 .

20. Cité par Ronald Steel, sp. cit. . p. 543 (notre traduction). 